\title{
Solution for GNSS height anomaly fitting of mining area based on robust TLS
}

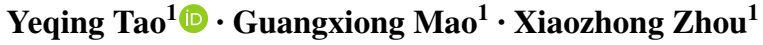

Received: 31 October 2017 / Accepted: 24 April 2018 / Published online: 14 May 2018

(C) Akadémiai Kiadó 2018

\begin{abstract}
Global navigation satellite system (GNSS) height solutions of mining area are readily contaminated by outliers because of the special geological environment. Additionally, GNSS height anomaly fitting model is a type of errors-in-variables model, and the traditional solution for parameter estimation does not account for error in the coefficient matrix. To solve these two problems, this paper presents a solution of the robust total least squares estimation for GNSS height anomaly fitting of mining area. Different from the traditional solution for robust estimation, an algorithm is established employing median method to obtain stable parameter values under the condition that observation data are highly contaminated. Employing Lagrange function and weight function, an iterative algorithm for the parameter estimation of GNSS anomaly fitting model is proposed, and the algorithm is verified using real data of mining area. The numerical results show that the proposed solution obtains stable parameter values when observation data are highly contaminated by outliers and demonstrate that the proposed algorithm is more accurate than traditional solutions for robust estimation.
\end{abstract}

Keywords Errors-in-variables model $\cdot$ Total least squares $\cdot$ Robust estimation $\cdot$ Median method $\cdot$ Height anomaly of mining area

\section{Introduction}

Height anomaly fitting is widely applied in mining area to make measurements using global navigation satellite system (GNSS) instead of making traditional measurements using leveling instrument. Height anomaly fitting is more difficult in mining area than in most other environments because unstable geological conditions and the complex terrain readily contaminate observation data with outliers. The traditional solution to processing survey data employs

Yeqing Tao

yenneytao@163.com

1 School of Urban and Environmental Sciences, Huaiyin Normal University, 111 Changjiang Road, Huaian 223300, People's Republic of China 
least-squares (LS) estimation to establish Gauss-Markov (GM) model, and provides an unbiased estimation when observation data contain only random error. However, parameter estimation using the traditional solution is biased if observation data are contaminated by outliers. Researchers have established feasible solutions with which to overcome the effects of outliers in observation data (Peter 1981; Jiangwen 1989; Yang 1999). It is noted that solutions to outliers are discussed usually in terms of the GM model, while the errors-in-variables (EIV) model is widely used in processing survey data. The EIV model is a model for which both the observation vector and coefficient matrix comprise observation data and therefore both elements have errors, while the GM model is considered a model for which only the observation vector has error. LS estimation is known to impose constraint on random error existing in the observation vector, and it cannot consider error existing in the observation vector and error existing in the coefficient matrix of the EIV model at the same time. Total least squares (TLS) estimation has been proposed to make an optimal estimation of parameters of the EIV model (Golub and Van Loan 1980; Schaffrin et al. 2006; Schaffrin and Felus 2008; Schaffrin and Wieser 2008; Mahboub 2012; Pan et al. 2015), and traditional problems of the GM model are discussed continuously under the EIV model, such as robust estimation (Mahboub et al. 2013; Xunqiang and Zhilin 2014; Tao et al. 2014), ill-posed problem (Xuming and Jicang 2012), model with equality or inequality constraints (Schaffrin 2006; Schaffrin and Felus 2009; Fang 2015).

Traditional solutions for robust LS estimation have been used to estimate EIV model parameters when outliers exist in observation data; e.g., the use of an equivalent weight function (Mahboub et al. 2013; Xunqiang and Zhilin 2014; Tao et al. 2014; Lu et al. 2014) and statistical testing (Schaffrin and Uzun 2011) are considered effective ways of robustly estimating EIV model parameters. However, current solutions have unavoidable shortcomings, which are discussed in the next section. As mentioned above, the EIV model is widely used in processing survey data. Although the topic of GNSS height anomaly fitting has been discussed extensively, it is noted that the GNSS height anomaly of mining area differs from that for other environments, while the fitting model is a type of EIV model and outliers exist in observation data of the fitting model. This paper presents a modified algorithm based on median method for robust TLS estimation and applies real data of height anomaly fitting in mining area to compare traditional solutions with the proposed solution and thus verify the feasibility of the proposed algorithm.

The remainder of the paper is organized as follows. Section 2 discusses traditional solutions for robust TLS estimation and analyzes their shortcomings. Section 3 discusses the solution of using the median method to obtain stable initial values of parameters when outliers exist in observation data, proposes a method of computing the weights of the observation vector and coefficient matrix, and presents an iterative algorithm for robust TLS estimation based on the median method. Section 4 applies the proposed iterative algorithm for height anomaly fitting of mining area, and uses real data of GNSS to verify the feasibility of the proposed algorithm and compare the accuracy of the proposed algorithm with that of traditional solutions. Finally, conclusions are drawn from the experimental results in Sect. 5. 


\section{Traditional solution for robust TLS estimation}

\subsection{GNSS height anomaly fitting model}

Elevation $(H)$ can be obtained by leveling measurement while the geodetic height $(h)$ can be obtained by GNSS measurement. Their difference is the height anomaly $(\delta)$ approximately, and there is no need to further discuss the reason for conducting height anomaly fitting work in the present paper. A quadric surface polynomial is taken as an effective model for GNSS height anomaly fitting (Heiskanen and Moritz 1967):

$$
\delta_{i}=b_{0}+b_{1} x_{i}+b_{2} y_{i}+b_{3} x_{i} y_{i}+b_{4} x_{i}^{2}+b_{5} y_{i}^{2}
$$

where $\delta_{i}$ is the height anomaly of observation points, $\left(x_{i}, y_{i}\right)$ denotes planar coordinates of observation points, and $\left(b_{0}, b_{1}, b_{2}, b_{3}, b_{4}, b_{5}\right)$ are the six parameters of the fitting model. Denoting the number of observations by $i(i>6)$, model (1) can be written as

$$
\left(\begin{array}{c}
\delta_{1} \\
\vdots \\
\delta_{i} \\
l
\end{array}\right)=\left(\begin{array}{cccccc}
1 & x_{1} & y_{1} & x_{1} y_{1} & x_{1}^{2} & y_{1}^{2} \\
\vdots & \vdots & \vdots & \vdots & \vdots & \vdots \\
1 & x_{i} & y_{i} & x_{i} y_{i} & x_{i}^{2} & y_{i}^{2}
\end{array}\right)\left(\begin{array}{c}
b_{0} \\
b_{1} \\
b_{2} \\
b_{3} \\
b_{4} \\
b_{5} \\
\boldsymbol{x}
\end{array}\right)
$$

where vector $\boldsymbol{l}$ is an observation vector comprising values of the height anomaly, matrix $\boldsymbol{B}$ is a coefficient matrix comprising planar coordinates or their function, and vector $\boldsymbol{x}$ comprises fitting model parameters. The vector $\boldsymbol{v}$ comprises error existing in observations that constitute vector $\boldsymbol{l}$, and this error follows a Gaussian distribution. The GM model is written as

$$
\left\{\begin{array}{l}
\boldsymbol{v}=\boldsymbol{B} \boldsymbol{x}-\boldsymbol{l} \\
\boldsymbol{v} \sim N\left(0, \sigma_{0}^{2} \boldsymbol{P}^{-1}\right)
\end{array}\right.
$$

where $\boldsymbol{P}$ is the weight matrix of the observation vector. Considering error in observation vector $\boldsymbol{l}$, model parameters (vector $\boldsymbol{x}$ ) can be computed according to LS estimation

$$
\left\{\begin{array}{l}
\boldsymbol{v}^{\mathrm{T}} \boldsymbol{P} \boldsymbol{v}=\min \\
\hat{\boldsymbol{x}}=\left(\boldsymbol{B}^{\mathrm{T}} \boldsymbol{P} \boldsymbol{B}\right)^{-1} \boldsymbol{B}^{\mathrm{T}} \boldsymbol{P} \boldsymbol{l}
\end{array}\right.
$$

The variance component $\sigma_{0}^{2}$ and the covariance matrix of parameters $\boldsymbol{D}(\boldsymbol{x})$ can be computed as

$$
\left\{\begin{array}{l}
\hat{\sigma}_{0}^{2}=\frac{\boldsymbol{v}^{\mathrm{T}} \boldsymbol{P} \boldsymbol{v}}{r} \\
\boldsymbol{D}(\hat{\boldsymbol{x}})=\hat{\sigma}_{0}^{2}\left(\boldsymbol{B}^{\mathrm{T}} \boldsymbol{P} \boldsymbol{B}\right)^{-1}
\end{array}\right.
$$

where $\boldsymbol{r}$ is the number of redundant observations. The solution for parameter estimation based on LS estimation simply implements the least constraint on error in the observation vector. However, elements of the coefficient matrix of the fitting model (Eq. 2) are also made up of observation data. In the GNSS height anomaly fitting model, therefore, the 
coefficient matrix also has error and the fitting model is an EIV model. The EIV model is written as

$$
\left\{\begin{array}{l}
\boldsymbol{v}=\left(\boldsymbol{B}+\boldsymbol{E}_{B}\right) \boldsymbol{x}-\boldsymbol{l} \\
{\left[\begin{array}{l}
\boldsymbol{v} \\
\boldsymbol{b}
\end{array}\right] \sim N\left(\left[\begin{array}{l}
0 \\
0
\end{array}\right], \sigma_{0}^{2}\left[\begin{array}{cc}
\boldsymbol{P}^{-1} & 0 \\
0 & \boldsymbol{P}_{\boldsymbol{B}}^{-1}
\end{array}\right]\right)}
\end{array}\right.
$$

where $\boldsymbol{E}_{\boldsymbol{B}}$ is a matrix of error that exists in matrix $\boldsymbol{B}, \boldsymbol{b}=\operatorname{vec}\left(\boldsymbol{E}_{\boldsymbol{B}}\right)$, and vec represents the conversion of a matrix to a column by stacking one column of the matrix underneath the previous column. $\boldsymbol{P}_{\boldsymbol{B}}$ is a weight matrix of the coefficient matrix. It is known that the traditional solution does not consider error in the coefficient matrix. To consider both error in the observation vector and error in the coefficient matrix and to apply the least constraint to them, TLS estimation is proposed as

$$
\boldsymbol{v}^{\mathrm{T}} \boldsymbol{P} \boldsymbol{v}+\boldsymbol{b}^{\mathrm{T}} \boldsymbol{P}_{\boldsymbol{B}} \boldsymbol{b}=\min
$$

\subsection{Discussion of traditional solutions of robust TLS estimation}

There are two solutions for processing outliers in observation data. One solution uses statistics theory to detach gross error while the other uses weight function to overcome the effect of gross error. The two methods are currently employed in the estimation of parameters of an EIV model with outliers. As previously mentioned, researchers are pursuing robust estimation based on weight function for the EIV model. Taking one algorithm based on the Lagrange function for EIV model estimation as an example, the estimation of parameter vector $\hat{\boldsymbol{x}}$, residual estimation of error vector $\hat{\boldsymbol{v}}$, and error vector $\hat{\boldsymbol{b}}$ can be obtained as (Xunqiang and Zhilin 2014)

$$
\begin{gathered}
\hat{\boldsymbol{x}}=-\left[\boldsymbol{A}_{0}^{\mathrm{T}} \boldsymbol{N}^{-1} \boldsymbol{A}_{0}\right]^{-1} \boldsymbol{A}_{0}^{\mathrm{T}} \boldsymbol{N}^{-1} \boldsymbol{\omega}^{0}+\boldsymbol{x}^{0} \\
{\left[\begin{array}{c}
\hat{\boldsymbol{v}} \\
\hat{\boldsymbol{b}}
\end{array}\right]=\left[\begin{array}{ll}
\boldsymbol{Q}_{1} & \boldsymbol{B}_{10}^{\mathrm{T}} \\
\boldsymbol{Q}_{2} & \boldsymbol{B}_{20}^{\mathrm{T}}
\end{array}\right] \hat{\boldsymbol{\lambda}}}
\end{gathered}
$$

where $\boldsymbol{A}_{0}$ is the partial derivative of the Lagrange function with respect to parameter vector $\boldsymbol{x}, \boldsymbol{x}^{0}$ is the parameter vector containing initial values, $\boldsymbol{\omega}^{0}=\boldsymbol{l}-\boldsymbol{B} \boldsymbol{x}^{0}$, and $\boldsymbol{N}$ is a function of $\boldsymbol{B}_{10}$ and $\boldsymbol{B}_{20}$, while $\boldsymbol{B}_{10}$ is the partial derivative of the Lagrange function with respect to error vector $\boldsymbol{v}$ and $\boldsymbol{B}_{20}$ is that with respect to error vector $\boldsymbol{b}$. $\hat{\lambda}$ is an estimation of the Lagrange multiplier vector, $\boldsymbol{Q}_{1}$ is the cofactor matrix of the observation vector, and $\boldsymbol{Q}_{2}$ is the cofactor matrix of the coefficient matrix. Detailed iteration steps of the algorithm can be found in the literature (Xunqiang and Zhilin 2014). Other algorithms that are extensively used have been described in seminal reports (Schaffrin and Felus 2008; Neitzel 2010; Xiaohua et al. 2011; Shen et al. 2011). The variance component $\sigma_{0}^{2}$ is estimated as

$$
\hat{\sigma}_{0}^{2}=\left(\boldsymbol{v}^{\mathrm{T}} \boldsymbol{P} \boldsymbol{v}+\boldsymbol{b}^{\mathrm{T}} \boldsymbol{P}_{\boldsymbol{B}} \boldsymbol{b}\right) / r
$$

It is noted that estimation of the variance component is biased when using current solutions (Shen et al. 2011; Mahboub 2014). According to the estimation of error vector $(\hat{\boldsymbol{v}}, \hat{\boldsymbol{b}})$, an iterative algorithm for robust TLS estimation based on weight function can be established. Taking the Huber weight function for example (Peter 1981), 


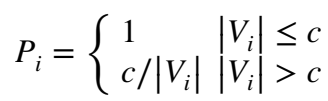

where $P_{\mathrm{i}}$ is the weight of observation data, $c$ is a threshold value in the range $2 \sigma_{0}-3 \sigma_{0}, V_{i}$ is the residual that exists in observation data and is estimation of error vector $(\hat{\boldsymbol{v}}, \hat{\boldsymbol{b}})$.

Robust estimation involves redefining the weights of observation data according to weight function and reducing the weights of observation data that include gross error through iteration. This process is considered to reduce the effect of gross error in observation data. Note that the solution for robust TLS estimation is similar to the traditional solution for robust LS estimation. Although the solution for robust TLS estimation that follows the traditional method has its advantages (e.g., it is easy to realize and its theory is mature), it has unavoidable disadvantages because the nature of the EIV model differs from that of the GM model.

In the GNSS height anomaly fitting of mining area, observation vector of the fitting model is made up of values of the height anomaly and the coefficient matrix of the model comprises planar coordinates or their function, and elements of the observation vector and coefficient matrix are thus obtained by different measurements and have different accuracy levels. However, current solutions for robust TLS estimation that are based on weight function use a unique $\sigma_{0}^{2}$ to determine the threshold of the weight function for observation data of the coefficient matrix and observation vector while the two types of data have different accuracy levels. Additionally, $\sigma_{0}^{2}$ computed by TLS estimation is biased (Schaffrin and Wieser 2008; Mahboub 2014). Therefore, the solution for robust estimation of the EIV model based on weight function obviously conflicts with the real condition of the fitting model as mentioned above. The threshold of the weight function computed separately for the observation vector and coefficient matrix is thus more rational. The problem of how to obtain separate $\sigma_{0}^{2}$ for the observation vector and coefficient matrix is discussed in the next section.

In LS estimation, the solution for parameter estimation based on TLS estimation implements the least constraint on error, which has the effect of averaging error into each observation variable, no matter whether the observation variables have error. Therefore, observation variables that are contaminated by gross error may not have a larger residual as supposed, and the solution of applying weight function for robust estimation may not have the supposed efficiency. In particular, in mining area, observation data are readily contaminated by gross error because of unstable geological conditions and complex terrain and, in the EIV model, both the observation vector and coefficient matrix could be contaminated by gross error. It is known that high contamination rate may lead to initial values of parameters deviating from true values, and an iterative algorithm for robust TLS estimation would be divergent. How to obtain a stable initial value when the EIV model is highly contaminated is discussed in the next section.

\section{Modified solution for robust TLS estimation}

Observation data are more readily contaminated by gross error in mining area than for other observation conditions, possibly resulting in parameter estimation deviating from true values. It is reasonable to believe that an optimal value is more difficult to obtain if the EIV model also exists at the same time. Least median of squares (LMS) regression is used to ensure initial values of parameters are stable (Rousseeuw and Wagner 1994) in the present study. Using Eq. (2), $C_{i}^{6}$ sets of parameter estimation can be obtained, and the median of parameters $\hat{\boldsymbol{x}}_{\text {med }}$ based on LMS regression is computed as 


$$
\left\{\begin{array} { l } 
{ b _ { 0 } ^ { 1 } b _ { 0 } ^ { 2 } \cdots b _ { 0 } ^ { C _ { i } ^ { 6 } } } \\
{ b _ { 1 } ^ { 1 } b _ { 1 } ^ { 2 } \cdots b _ { 1 } ^ { C _ { i } ^ { 6 } } } \\
{ b _ { 2 } ^ { 1 } b _ { 2 } ^ { 2 } \cdots b _ { 2 } ^ { C _ { i } ^ { 6 } } } \\
{ b _ { 3 } ^ { 1 } b _ { 3 } ^ { 2 } \cdots b _ { 3 } ^ { C _ { i } ^ { 6 } } } \\
{ b _ { 4 } ^ { 1 } b _ { 4 } ^ { 2 } \cdots b _ { 4 } ^ { C _ { i } ^ { 6 } } } \\
{ b _ { 5 } ^ { 1 } b _ { 5 } ^ { 2 } \cdots b _ { 5 } ^ { C _ { i } ^ { 6 } } }
\end{array} \quad \left\{\begin{array}{l}
\operatorname{med}\left(b_{0}\right)=\operatorname{median}\left(b_{0}^{1} b_{0}^{2} \cdots b_{0}^{C_{i}^{6}}\right) \\
\operatorname{med}\left(b_{1}\right)=\operatorname{median}\left(b_{1}^{1} b_{1}^{2} \cdots b_{1}^{C_{i}^{6}}\right) \\
\operatorname{med}\left(b_{2}\right)=\operatorname{median}\left(b_{2}^{1} b_{2}^{2} \cdots b_{2}^{C_{i}^{6}}\right) \\
\operatorname{med}\left(b_{3}\right)=\operatorname{median}\left(b_{3}^{1} b_{3}^{2} \cdots b_{3}^{C_{i}^{6}}\right) \\
\operatorname{med}\left(b_{4}\right)=\operatorname{median}\left(b_{4}^{1} b_{4}^{2} \cdots b_{4}^{C_{i}^{6}}\right) \\
\operatorname{med}\left(b_{5}\right)=\operatorname{median}\left(b_{5}^{1} b_{5}^{2} \cdots b_{5}^{C_{i}^{6}}\right)
\end{array} \rightarrow \hat{x}_{\operatorname{med}}=\left[\begin{array}{l}
\operatorname{med}\left(b_{0}\right) \\
\operatorname{med}\left(b_{1}\right) \\
\operatorname{med}\left(b_{2}\right) \\
\operatorname{med}\left(b_{3}\right) \\
\operatorname{med}\left(b_{4}\right) \\
\operatorname{med}\left(b_{5}\right)
\end{array}\right]\right.\right.
$$

In a seminal report (Yang 1999), by LMS regression, stable values of parameters were obtained while the contamination rate of gross error in observation data was not more than 50\%, and LMS regression is thus concluded to be suitable for processing survey data of a mining area. Using initial values of parameters $\left(\hat{\boldsymbol{x}}_{\text {med }}\right)$, the solution for parameter estimation of the EIV model is applied to estimate error in the observation coefficient and observation vector. Note that the used solution should consider the stochastic character of the model, and the present study employs an algorithm based on Lagrange function (Xunqiang and Zhilin 2014). The estimation of error in the observation vector $(\hat{\boldsymbol{v}})$ and coefficient matrix $(\hat{\boldsymbol{b}})$ is

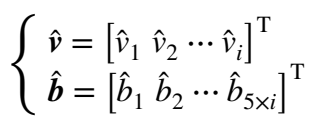

As discussion in the previous section, traditional solutions for robust TLS estimation are based on weight function, and the threshold value of the weight function for both the coefficient matrix and observation vector is determined by variance component $\sigma_{0}^{2}$. To overcome shortcomings of traditional solutions, a threshold value of the weight function is computed separately for the coefficient matrix and observation vector. LMS regression still needs to be used to compute the median of error estimation, and respective variance components are computed as

$$
\left\{\begin{array} { l } 
{ \hat { v } _ { \text { med } } = \operatorname { m e d i a n } ( \hat { \boldsymbol { v } } ) } \\
{ \hat { b } _ { \text { med } } = \operatorname { m e d i a n } ( \hat { \boldsymbol { b } } ) }
\end{array} \rightarrow \left\{\begin{array}{l}
\sigma_{l}=1.483 \hat{v}_{\text {med }} \\
\sigma_{b}=1.483 \hat{b}_{\text {med }}
\end{array}\right.\right.
$$

According to the LMS and Lagrange algorithm for TLS estimation, an iterative algorithm for height anomaly fitting, especially in the case of highly contaminated survey data of mining area, is established as follows.

Step 1 The LMS and Lagrange algorithm is used to compute initial values of parameters $\left(\hat{\boldsymbol{x}}_{\text {med }}\right)$

Step 2 From the median estimation of parameters $\left(\hat{\boldsymbol{x}}_{\text {med }}\right)$, error in the coefficient matrix and observation vector is estimated (Eq. 9)

Step 3 Weight function is used to redefine the weight of observation data, and the threshold of the weight function is computed using respective variance components $\left(\sigma_{l}, \sigma_{b}\right)$ 
Table 1 Iterative algorithm of LMS-RTLS

1. Initial values of parameters calculation $\left\{\begin{array}{c}\hat{\boldsymbol{x}}_{1}^{0}=-\left[\boldsymbol{A}_{0}^{\mathrm{T}} \boldsymbol{N}^{-1} \boldsymbol{A}_{0}\right]^{-1} \boldsymbol{A}_{0}^{\mathrm{T}} \boldsymbol{N}^{-1} \boldsymbol{\omega}^{0}+\boldsymbol{x}^{0} \\ \vdots \\ \hat{\boldsymbol{x}}_{C_{i}^{6}}^{0}=-\left[\boldsymbol{A}_{0}^{\mathrm{T}} \boldsymbol{N}^{-1} \boldsymbol{A}_{0}\right]^{-1} \boldsymbol{A}_{0}^{\mathrm{T}} \boldsymbol{N}^{-1} \boldsymbol{\omega}^{0}+\boldsymbol{x}^{0}\end{array} \rightarrow \hat{\boldsymbol{x}}_{\mathrm{med}}\right.$

2. Utilization $\hat{\boldsymbol{x}}_{\text {med }}$ for calculation variance components $\left(\sigma_{l}, \sigma_{b}\right)$ by median function

$$
\left[\begin{array}{l}
\hat{\boldsymbol{v}} \\
\hat{\boldsymbol{b}}
\end{array}\right]=\left[\begin{array}{ll}
\boldsymbol{Q}_{1} & \boldsymbol{B}_{10}^{\mathrm{T}} \\
\boldsymbol{Q}_{2} & \boldsymbol{B}_{20}^{\mathrm{T}}
\end{array}\right] \hat{\boldsymbol{\lambda}} \rightarrow\left\{\begin{array} { l } 
{ \hat { v } _ { \text { med } } = \operatorname { m e d i a n } ( \hat { \boldsymbol { v } } ) } \\
{ \hat { b } _ { \text { med } } = \operatorname { m e d i a n } ( \hat { \boldsymbol { b } } ) }
\end{array} \rightarrow \left\{\begin{array}{l}
\sigma_{l}=1.483 \hat{v}_{\text {med }} \\
\sigma_{b}=1.483 \hat{b}_{\text {med }}
\end{array}\right.\right.
$$

3. Robust TLS estimation by iteration based on weight function, note that, threshold values of weight functions are defined respectively by $\left(\sigma_{l}, \sigma_{b}\right)$ for the two kinds of observation data

$P_{i}= \begin{cases}1 & \left|V_{i}\right| \leq c \\ c /\left|V_{i}\right| & \left|V_{i}\right|>c\end{cases}$

4. $C_{i}^{6}$ sets of estimation $\left(\hat{\boldsymbol{x}}_{1} \hat{\boldsymbol{x}}_{2} \cdots \hat{\boldsymbol{x}}_{C^{6}}\right)$ can be obtained at most by step 2 to step 3 , and Optimal estimation for model parameters can be chosen from the sets by minimum-norm criterion

$\min \left(\left\|\hat{\boldsymbol{x}}_{1}-\hat{\boldsymbol{x}}_{\text {med }}\right\|,\left\|\hat{\boldsymbol{x}}_{2}-\hat{\boldsymbol{x}}_{\text {med }}\right\|, \cdots,\left\|\hat{\boldsymbol{x}}_{C_{i}^{6}}-\hat{\boldsymbol{x}}_{\text {med }}\right\|\right)$

Step 4 By redefining the weight of observation data, the final estimation of parameters can be obtained by iteration from step 2 to step 3. $C_{i}^{6}$ sets of estimation can be obtained $\left(\hat{\boldsymbol{x}}_{1} \hat{\boldsymbol{x}}_{2} \cdots \hat{\boldsymbol{x}}_{C_{i}^{6}}\right)$, and the optimal estimation of parameters can be chosen from estimation sets using a special criterion, such as the minimum-norm criterion or minimum-variance-component criterion

This presented algorithm is named LMS-RTLS, the algorithm is presented step by step in Table 1.

The efficiency of this algorithm is compared with that of traditional robust TLS (R-TLS) estimation using real data of mining area in the next section.

\section{Experimental example and analysis}

An experiment is carried out to verify the accuracy of data obtained in GNSS control survey for mining area. Coordinates of control points obtained by the GNSS and known coordinates of points in the mining area are listed in Table 2.

Table 2 Results of checking planar coordinates of control points

\begin{tabular}{llllll}
\hline Dot mark & \multicolumn{2}{l}{ Known coordinates } & & \multicolumn{2}{l}{ Coordinates by GNSS } \\
\cline { 2 - 3 } \cline { 6 - 7 } & $x_{1}(\mathrm{~m})$ & $y_{1}(\mathrm{~m})$ & & $x_{2}(\mathrm{~m})$ & $y_{2}(\mathrm{~m})$ \\
\hline 1 & 3909.484 & 653.075 & & 3909.484 & 653.075 \\
2 & 3892.751 & 919.480 & & 3892.883 & 919.342 \\
3 & 4297.145 & 920.001 & & 4297.131 & 920.133 \\
\hline
\end{tabular}

For coordinates obtained by the GNSS, the coordinates of control point No. 1 are taken as true values while coordinates of other points are obtained by constraint estimation 
Table 3 Relative position differences of control points

\begin{tabular}{|c|c|c|c|c|c|c|}
\hline \multirow[t]{2}{*}{ Dot mark } & \multicolumn{2}{|c|}{ Known coordinates } & \multicolumn{2}{|c|}{ Coordinates by GNSS } & \multicolumn{2}{|c|}{ Relative difference } \\
\hline & Distance (m) & Azimuth & $\overline{\text { Distance }(\mathrm{m})}$ & Azimuth & Distance & Azimuth \\
\hline $1-2$ & 266.930 & $93^{\circ} 35^{\prime} 38.57^{\prime \prime}$ & 266.784 & $93^{\circ} 34^{\prime} 03.07^{\prime \prime}$ & $1 / 1828$ & $1^{\prime} 35.50^{\prime \prime}$ \\
\hline $2-3$ & 404.394 & $0^{\circ} 04^{\prime} 25.74^{\prime \prime}$ & 404.249 & $0^{\circ} 06^{\prime} 43.55^{\prime \prime}$ & $1 / 2786$ & $2^{\prime} 17.81^{\prime \prime}$ \\
\hline
\end{tabular}

Differences in distances and azimuths between coordinates from Table 2 are computed and listed in Table 3 . The results of verification demonstrate that observation data of the mining area are more readily contaminated by gross error than observation data obtained for other conditions.

In the next part, observation data of the mining area are used to compare the proposed solution (LMS-RTLS) with traditional solutions. The observation data obtained by the GNSS and leveling measurements are listed in Table 4.

Table 4 Observation data obtained by the GNSS and leveling measurements in the mining area

\begin{tabular}{llllll}
\hline Dot mark & $y(\mathrm{~m})$ & $x(\mathrm{~m})$ & $H(\mathrm{~m})$ & $h(\mathrm{~m})$ & $\delta(\mathrm{m})$ \\
\hline 1 & 8240.891 & 7063.584 & 1218.084 & 1073.383 & -144.701 \\
2 & 8636.185 & 6924.577 & 1210.865 & 1066.166 & -144.699 \\
3 & 8622.459 & 6685.552 & 1153.315 & 1008.603 & -144.712 \\
4 & 8591.174 & 6419.837 & 1129.854 & 985.143 & -144.711 \\
5 & 8174.893 & 6514.292 & 1129.431 & 984.724 & -144.707 \\
6 & 8554.675 & 6122.861 & 1160.549 & 1015.85 & -144.699 \\
7 & 8554.675 & 5893.616 & 1181.939 & 1037.242 & -144.697 \\
8 & 9056.177 & 5727.623 & 1189.669 & 1044.991 & -144.678 \\
9 & 9029.507 & 6045.128 & 1170.579 & 1025.897 & -144.682 \\
10 & 9062.052 & 6273.595 & 1154.21 & 1009.517 & -144.693 \\
11 & 9455.693 & 5950.708 & 1189.202 & 1044.542 & -144.66 \\
12 & 9045.588 & 6520.762 & 1190.983 & 1046.299 & -144.684 \\
13 & 9038.551 & 6778.472 & 1185.02 & 1040.327 & -144.693 \\
14 & 9414.566 & 6614.093 & 1153.934 & 1009.243 & -144.691 \\
15 & 9007.187 & 7048.716 & 1192.049 & 1047.359 & -144.69 \\
16 & 8624.444 & 7105.879 & 1248.524 & 1103.832 & -144.692 \\
17 & 8598.000 & 7242.000 & 1253.016 & 1108.321 & -144.695 \\
18 & 9029.507 & 7301.472 & 1201.148 & 1056.459 & -144.689 \\
19 & 9431.254 & 7100.754 & 1203.835 & 1059.153 & -144.682 \\
20 & 8174.893 & 5915.484 & 1186.202 & 1041.5 & -144.702 \\
21 & 8559.889 & 5674.792 & 1202.177 & 1057.5 & -144.677 \\
22 & 9066.705 & 5495.013 & 1233.633 & 1088.963 & -144.67 \\
23 & 8559.889 & 5482.018 & 1238.359 & 1093.682 & -144.677 \\
24 & 8113.515 & 5319.615 & 1232.894 & 1088.21 & -144.684 \\
25 & 8555.847 & 5142.927 & 1225.299 & 1080.635 & -144.664 \\
26 & 9089.025 & 5234.824 & 1174.662 & 1030.012 & -144.65 \\
27 & 9453.573 & 5450.409 & 1176.093 & 1031.462 & -144.631 \\
\hline & & & & & \\
10 & & & & &
\end{tabular}




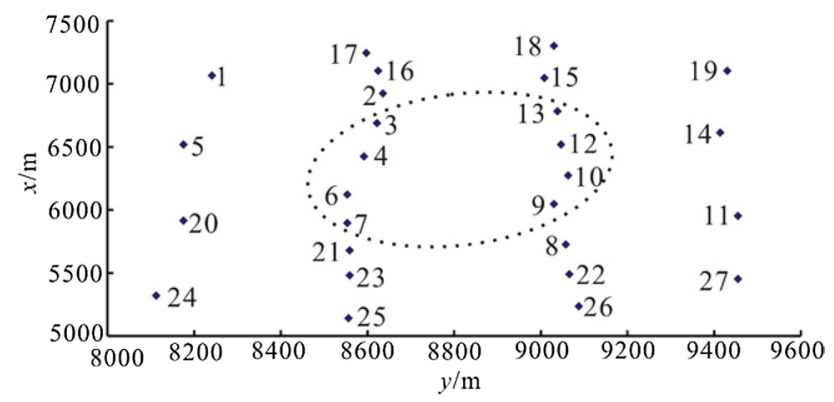

Fig. 1 Distribution of control points

Table 5 Data of control points with gross error

Table 6 Fitting residuals of control points

\begin{tabular}{llll}
\hline Dot mark & $y(\mathrm{~m})$ & $x(\mathrm{~m})$ & $\delta(\mathrm{m})$ \\
\hline 4 & 8593.374 & 6419.837 & -144.711 \\
7 & 8554.675 & 5893.616 & -146.697 \\
10 & 9062.052 & 6272.095 & -144.693 \\
13 & 9038.551 & 6778.472 & -145.693 \\
\hline
\end{tabular}

\begin{tabular}{lcccc}
\hline Dot mark & LS $(\mathrm{m})$ & TLS $(\mathrm{m})$ & LS $(\mathrm{g} / \mathrm{m})$ & TLS $(\mathrm{g} / \mathrm{m})$ \\
\hline 3 & -0.016 & -0.002 & 0.137 & 0.032 \\
4 & -0.021 & 0.006 & -0.193 & -0.220 \\
6 & -0.015 & -0.005 & -0.024 & 0.463 \\
7 & -0.013 & 0.002 & 0.061 & -0.241 \\
9 & -0.024 & 0.00005 & -0.013 & 0.218 \\
10 & 0.001 & 0.0006 & -0.172 & -0.278 \\
12 & -0.030 & -0.002 & 0.372 & 0.087 \\
13 & -0.016 & 0.0009 & -0.212 & 0.076 \\
\hline
\end{tabular}

Control points Nos. 3, 4, 6, 7, 9, 10, 12, and 13 are used to compute parameters of the fitting model using three algorithms. The distribution of control points is shown in Fig. 1.

The quantity of observation data for computation should be more than the quantity needed to obtain the variance of parameter estimation. The fitting model (2) requires six sets of observation data for computing parameters, and a maximum of eight sets of parameter estimation can be computed using the eight control points. Error of 1-3 $\mathrm{m}$ is introduced to coordinates of control points Nos. 4, 7, 10, and 13 while error of 1-2 $\mathrm{m}$ is introduced to the corresponding height anomalies to simulate gross error. Data are listed in Table 5 (where data with gross error are indicated in italics).

Control points for the computation of model parameters that have not been contaminated with gross error are firstly used to compute parameters based on LS and TLS estimation. It is noted that the condition number of the normal matrix is $1.26 \times 10^{24}$, the normal equation is ill posed, and ridge estimation is used for regularization (Dongfang et al. 2016). Fitting residuals of control points based on LS and TLS algorithms (based 
Table 7 Accuracy of the fitting model determined by check points

\begin{tabular}{lrrrr}
\hline Dot mark & LS $(\mathrm{m})$ & TLS $(\mathrm{m})$ & LS $(\mathrm{g} / \mathrm{m})$ & TLS $(\mathrm{g} / \mathrm{m})$ \\
\hline 1 & 0.660 & -0.441 & 1.719 & 2.257 \\
2 & 0.001 & -0.038 & -1.547 & 0.287 \\
5 & 0.685 & -0.422 & 4.946 & 2.687 \\
8 & 0.048 & -0.036 & -2.298 & -1.436 \\
11 & 0.734 & -0.429 & 4.184 & 4.503 \\
14 & 0.573 & -0.295 & 2.654 & 1.346 \\
15 & -0.012 & -0.012 & -4.146 & -4.580 \\
16 & 0.044 & -0.079 & -3.360 & -3.017 \\
17 & 0.108 & -0.116 & -4.925 & -2.347 \\
18 & 0.048 & -0.045 & -8.059 & -5.290 \\
19 & 0.610 & -0.322 & -2.975 & -3.325 \\
20 & 0.609 & -0.350 & 1.014 & 2.049 \\
21 & -0.021 & -0.013 & -4.640 & -3.915 \\
22 & 0.105 & -0.072 & -5.162 & -5.225 \\
23 & 0.003 & -0.015 & -7.728 & -7.013 \\
24 & 0.757 & -0.414 & -8.851 & -9.056 \\
25 & 0.059 & -0.045 & -14.815 & -12.939 \\
26 & 0.189 & -0.140 & -9.382 & -7.892 \\
27 & 0.843 & -0.529 & -0.352 & 1.057 \\
\hline
\end{tabular}

Table 8 Residual existing in observation data of the coefficient matrix

\begin{tabular}{lrrrrr}
\hline Dot mark & \multicolumn{1}{l}{$x$} & \multicolumn{1}{c}{$x y$} & \multicolumn{1}{c}{$x^{2}$} & \multicolumn{1}{c}{$y^{2}$} \\
\hline 3 & -0.004 & 0.004 & $1.84-07$ & $1.84 \mathrm{E}-07$ & $-2.95 \mathrm{E}-07$ \\
4 & 0.024 & -0.025 & $-1.13 \mathrm{E}-06$ & $-1.13 \mathrm{E}-06$ & $1.80 \mathrm{E}-06$ \\
6 & -0.053 & 0.053 & $2.43 \mathrm{E}-06$ & $2.43 \mathrm{E}-06$ & $-3.89 \mathrm{E}-06$ \\
7 & 0.027 & -0.027 & $-1.23 \mathrm{E}-06$ & $-1.23 \mathrm{E}-06$ & $1.97 \mathrm{E}-06$ \\
9 & -0.025 & 0.025 & $1.16 \mathrm{E}-06$ & $1.16 \mathrm{E}-06$ & $-1.85 \mathrm{E}-06$ \\
10 & 0.031 & -0.031 & $-1.43 \mathrm{E}-06$ & $-1.43 \mathrm{E}-06$ & $2.28 \mathrm{E}-06$ \\
12 & -0.010 & 0.010 & $4.75 \mathrm{E}-07$ & $4.75 \mathrm{E}-07$ & $-7.60 \mathrm{E}-07$ \\
13 & -0.009 & 0.009 & $4.29 \mathrm{E}-07$ & $4.28 \mathrm{E}-07$ & $-6.85 \mathrm{E}-07$ \\
\hline
\end{tabular}

on singular value decomposition, Golub and Van Loan 1980) are listed in Table 6, marked LS and TLS respectively. To compare with observation data that are free of gross error, control points of which some are contaminated with gross error (listed in Table 5) are used to compute parameters on the basis of LS and TLS estimation, which have 100 iterations. These results are also listed in Table 6, marked LSg and TLSg respectively.

Control points not used to compute model parameters are used to check the accuracy of the fitting model. The results are listed in Table 7.

Comparison with computation results obtained using control points that are free of gross error shows that the introduction of gross error into control points clearly affects the results of parameter estimation. To overcome the effect of gross error on parameter estimation, robust estimation is used for parameter estimation. Two algorithms-traditional 
Table 9 Fitting residuals of observation data obtained by LMS-RTLS

\begin{tabular}{lccccc}
\hline Dot mark & Residual $(\mathrm{m})$ & Dot mark & Residual $(\mathrm{m})$ & Dot mark & Residual $(\mathrm{m})$ \\
\hline 3 & 0.091 & 2 & -0.043 & 19 & 0.128 \\
4 & 0.079 & 5 & 0.105 & 20 & 0.084 \\
6 & -0.006 & 8 & 0.596 & 21 & -1.040 \\
7 & 0.024 & 11 & -0.267 & 22 & 0.923 \\
9 & -0.024 & 14 & 1.027 & 23 & -0.220 \\
10 & 0.039 & 15 & -0.258 & 24 & 0.181 \\
12 & -0.084 & 16 & -0.467 & 25 & 0.206 \\
13 & -0.009 & 17 & 0.151 & 26 & 0.575 \\
1 & 0.281 & 18 & 0.187 & 27 & -0.648 \\
\hline
\end{tabular}

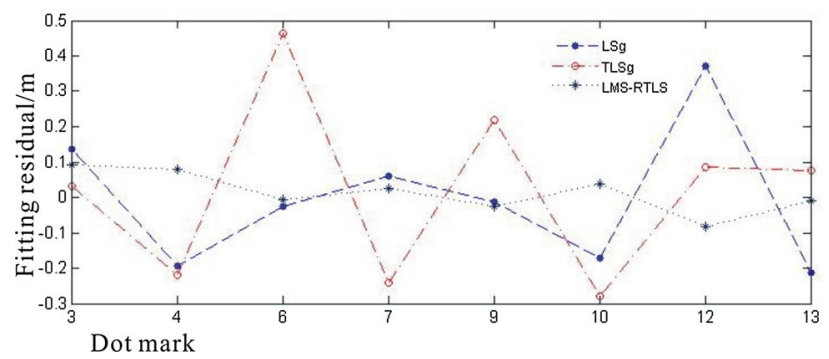

Fig. 2 Distribution of the fitting residual for control points

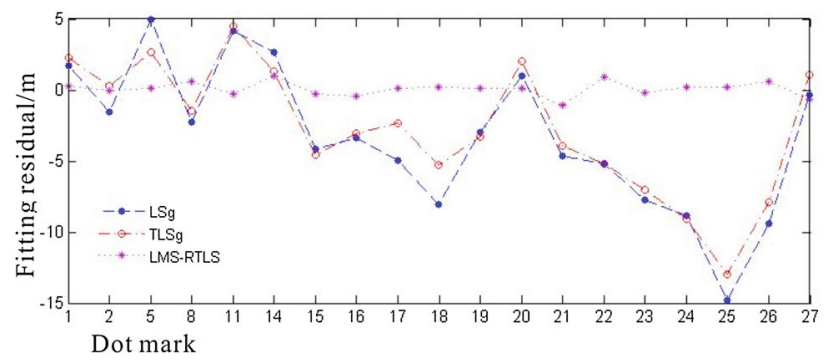

Fig. 3 Distribution of the fitting residual for check control points

robust TLS estimation (R-TLS) and the proposed algorithm (LMS-RTLS) - are used for parameter estimation of the fitting model with 100 iterations, and the fitting residual in observation data of the coefficient matrix is listed in Table 8 (Schaffrin and Wieser 2008).

The variance component $\sigma_{0}$ is 0.474 (computed by the proposed algorithm of Schaffrin and Wieser 2008). It is known that the residual estimation of elements in an observation 
vector and coefficient matrix (listed in Tables 7 and 8) is smaller than the variance component, and robust TLS estimation thus fails to optimally estimate model parameters. The reason why the residual estimation of observation data is smaller than the variance component has been explained in the literature (Xiaohua et al. 2011). To apply the proposed algorithm in the present study, the eight control points (Nos. 3, 4, 6, 7, 9, 10, 12, and 13) are separated into eight groups, with each group having seven control points. Applying the LMS-RTLS algorithm, the fitting residual of the eight control points and check control points is computed as listed in Table 9.

Figure 2 shows the distribution of fitting residuals for control points computed separately by LS estimation, R-TLS estimation, and LMS-RTLS estimation while Fig. 3 shows the distribution of fitting residuals for check control points.

Results reveal that traditional solutions fail in robust and robust estimation; i.e., the EIV model based on LMS regression is more efficient than traditional robust estimation when observation data have gross error. Observation data are more readily contaminated by gross error in a mining area than under usual conditions. The results show that gross error in the observation data obviously and adversely affects the final accuracy of the height anomaly fitting. Additionally, TLS estimation is less efficient than LS estimation when observation data have gross error while the fitting result based on TLS estimation is clearly more accurate than that based on LS estimation when observation data are free of gross error. Note that, because of unavoidable shortcomings, the traditional robust solution fails in estimation. Furthermore, the height anomaly fitting model is an ill-posed model, which obviously affects the fitting accuracy.

\section{Conclusions}

There is an extensive robust estimation problem in the field of geodesy. This paper mainly addresses robust TLS estimation for GNSS height anomaly fitting of mining area, which obviously has an EIV model. An analysis of traditional solutions reveals two obvious disadvantages of current solutions, which are verified experimentally. To overcome the shortcomings of traditional solutions for parameter estimation of an EIV model with outliers, a modified algorithm based on LMS regression was proposed. The proposed solution uses the median method to compute stable initial values and computes separate threshold values of the weight function for the coefficient matrix and observation vector. In an experiment, an iterative algorithm based on the proposed solution was demonstrated to be feasible and shown to obtain results that were more stable and accurate than traditional methods. Additionally, its efficiency was compared with the efficiencies of traditional solutions. The experiment revealed that traditional robust TLS estimation has no clear advantage over LS estimation of mining area when observation data have gross error.

Acknowledgements The authors would like to thank the reviewers and the editor. This research was supported by the National Natural Science Foundation of China (41601501; 41271135), Natural Science Found for Colleges and Universities of Jiangsu Province (16KJD420001) and Huaian Key Laboratory of Geographic Information Technology and Applications (HAP201405). 


\section{References}

Adcock RJ (1877) Note on the method of least squares. Analyst 4:183-184

Amiri-Simkooei AR (2013) Application of least squares variance component estimation to errors-in-variables models. J Geodesy 87(10):935-944

Dongfang L, Jianjun Z, Yingchun S et al (2016) Construction method of regularization by singular value decomposition of design matrix. Acta Geod Cartogr Sin 45(8):883-889

Fang X (2015) Weighted total least-squares with constraints: a universal formula for geodetic symmetrical transformations. J Geodesy 89(5):459-469

Golub GH, Van Loan CF (1980) An analysis of the total least squares problem. SIAM J Number Anal 17:883-893

Heiskanen WA, Moritz H (1967) Physical geodesy. W H Freeman and Company, San Francisco

Jiangwen Z (1989) Classic theory of errors and robust estimation. Acta Geod Cartogr Sin 18(2):115-120

Ling Y, Yunzhong S, Lizhi L (2011) Equivalent weight robust estimation method based on median parameter estimates. Acta Geod Cartogr Sin 40(1):28-32

Lu J, Chen Y, Li BF, Fang X (2014) Robust total least squares with reweighting iteration for three-dimensional similarity transformation. Surv Rev 46(334):28-36

Mahboub V (2012) On weighted total least-squares for geodetic transformation. J Geodesy 86(5):359-367

Mahboub V (2014) Variance component estimation in errors-in-variables models and a rigorous total leastsquares approach. Stud Geophys Geod 58(1):17-40

Mahboub V, Amiri-Simkooei AR, Sharifi MA (2013) Iteratively reweighted total least squares: a robust estimation in error-in-variables models. Surv Rev 45(329):92-99

Neitzel F (2010) Generalization of total least-squares on example of unweighted and weighted 2D similarity transformation. J Geodesy 84(12):751-762

Pan G, Zhou Y, Sun H et al (2015) Linear observation based total least squares. Surv Rev 47(340):18-27

Peiliang X, Liu J, Shi C (2012) Total least squares adjustment in partial errors- in-variables models: algorithm and statistical analysis. J Geodesy 86(8):661-675

Peter JH (1981) Robust statistics. Wiley, Hoboken

Rousseeuw P, Wagner J (1994) Robust regression with a distribution intercept using least median of squares. Comput Stat Data Anal 17:65-76

Schaffrin B (2006) A note on constrained total least-squares estimation. Linear Algebra Appl 417:245-258

Schaffrin B, Felus YA (2008) On the multivariate total least-squares approach to empirical coordinate transformations: three algorithms. J Geodesy 82(6):373-383

Schaffrin B, Felus YA (2009) An algorithmic approach to the total least-squares problem with linear and quadratic constraints. Stud Geophys Geod 53:1-16

Schaffrin B, Uzun S (2011) Error-in-variables for mobile mapping algorithms in the presence of outliers. Arch Photogramm Cartogr Remote Sens 22:377-387

Schaffrin B, Wieser A (2008) On weighted total least-squares adjustment for linear regression. J Geodesy 82(7):415-421

Schaffrin B, Lee I, Choi Y et al (2006) Total least squares (TLS) for geodetic straight-line and plane adjustment. Bull Geod Sc Aff 65:141-168

Shen Y, Li B, Chen Y (2011) An iterative solution of weighted total least-squares adjustment. J Geodesy 85(4):229-238

Tao Y, Gao J, Yao Y (2014) TLS algorithm for GPS height fitting based on robust estimation. Surv Rev 46(336):184-188

Xiaohua T, Yanmin J, Lingyun L (2011) An improved weighted total least squares method with applications in linear fitting and coordinate transformation. J Surv Eng 137(4):120-128

Xuming G, Jicang W (2012) Generalized regularization to ill-posed total least squares problem. Acta Geod Cartogr Sin 41(3):372-377

Xunqiang G, Zhilin L (2014) A robust weighted total least squares method. Acta Geod Cartogr Sin 43(9):888-894

Yang Y (1999) Robust estimation of geodetic datum transformation. J Geodesy 73(5):268-274 